\title{
Nonsyndromic Oligodontia Involving the Maxillary Arch: A Rare Case Report
}

\author{
Pawandeep KS Virk ${ }^{1}$, Urvashi Sharma²
}

\begin{abstract}
A clinical case of a 12-year-old girl involving agenesis of multiple permanent maxillary teeth, and presence of a full complement of permanent mandibular teeth, is reported. No such case is previously reported in literature. A prosthetic rehabilitation using a removable maxillary denture was performed to improve the facial profile, have satisfactory intercuspation of teeth, and restore masticatory function along with significant esthetic improvement in smile. Careful monitoring on a long-term basis, educating the parents about growth changes in the child and importance of replacing prostheses according to these changes, is desired.

Keywords: Child, Denture, Esthetics, Maxilla, Partial.

Journal of South Asian Association of Pediatric Dentistry (2021): 10.5005/jp-journals-10077-3053
\end{abstract}

\section{INTRODUCTION}

Agenesis of multiple teeth is rare. Synonyms for missing teeth include hypodontia, oligodontia, and anodontia. Oligodontia denotes a stronger clinical expression of hypodontia, has a wider and a more nonspecific definition. Schalk van der Weide defines oligodontia as hypodontia of six or more permanent teeth, except third molar. ${ }^{1}$ Oligodontia can occur either as an isolated anomaly (oligodontia-I) or as part of a syndrome (oligodontia-S), sometimes associated with as many as 120 syndromes. ${ }^{2,3}$ The numerous variants of oligodontia can be identified by other associated features of syndromes.

The prevalence of oligodontia is $0.3 \%$ in permanent teeth and less in primary teeth $0.1-0.9 \% .^{4,5}$ The occurrence is more common in females than males in the ratio of $3: 2 .^{3,4}$ The etiology of oligodontia is unclear; several theories have been proposed, such as trauma during odontogenesis, disorders of endocrine system, infections, radiation or chemotherapy for cancers occurring in the early years of life. ${ }^{2}$ However, role of genetics is recently corroborated by investigators. ${ }^{4,6}$

Oligodontia can affect the growth of maxilla and mandible, compromising facial esthetics. Other dental anomalies associated are the reduction in size and form of teeth, delayed formation, eruption and malposition of teeth, taurodontism, and enamel hypoplasia., $\mathrm{A}$ change in the facial appearance and speech impairment has a bearing on the physiological and psychosocial impact on the individual, resulting in low self-confidence and social acceptance. An early diagnosis and treatment can improve appearance, functions of mastication and speech while simultaneously decreasing the damaging psychosocial effects on the child. ${ }^{3,5}$

The present case describes the prosthetic rehabilitation of a case of oligodontia involving a complete absence of maxillary teeth except for the presence of tooth 16 (permanent maxillary right first molar) and an anomalous tooth 55 (primary maxillary right second molar) in a 12-year-old girl.

\section{Case Description}

A 12-year-old girl reported for esthetic dental rehabilitation of multiple missing teeth in the upper jaw. A detailed clinical history
${ }^{1}$ Pediatric Dentist, Virk Dental Clinic, Ludhiana, Punjab, India

${ }^{2}$ Department of Pedodontics and Preventive Dentistry, Dr HSJ Institute of Dental Sciences and Hospital, Chandigarh, India

Corresponding Author: Pawandeep KS Virk, Ludhiana, Punjab, India, Phone: +91 9888499845, e-mail: psandhuvirk@yahoo.com

How to cite this article:Virk PKS, Sharma U. Nonsyndromic Oligodontia Involving the Maxillary Arch: A Rare Case Report. J South Asian Assoc Pediatr Dent 2021;4(1):54-57.

Source of support: Nil

Conflict of interest: None

was recorded with the parent and patient. There was no significant medical history. The family history revealed that she was born to nonconsanguineous parents with no history of missing teeth in any family member. There was no history of trauma, extraction, or premature exfoliation of these primary teeth. The dental history revealed that the child had visited a dentist when she was 5 -yearsold with a complaint of multiple missing teeth. The dentist had then, inserted a removable maxillary denture which the patient had been wearing ever since, without replacement. The patient was presently, not happy with the prosthesis and wanted to replace it for esthetic and functional reasons. No abnormality was evident on general physical examination. The height and the weight of the patient were within normal limits, she was interactive and mentally sound.

The extraoral clinical examination showed a slight concave profile (Fig. 1), lack of upper lip support with a good lip seal, procumbent lower lip contour, and an acceptable facial symmetry. On palpation of the temporomandibular joint, no clicking or crepitus was detected, and no sensitivity of masticatory muscles was observed. There was no speech problem in the patient. An intraoral examination revealed the complete absence of all maxillary teeth except for the presence of tooth 16 and an anomalous, rotated tooth 55 (Fig. 2). The maxillary arch displayed micrognathia with a hypoplastic alveolar ridge. The mandibular arch was well formed with all complement of teeth present (Fig. 3). An initial removable partial denture worn by the patient since the age of 5 years was ill-fitting and poorly designed (Fig. 4). 


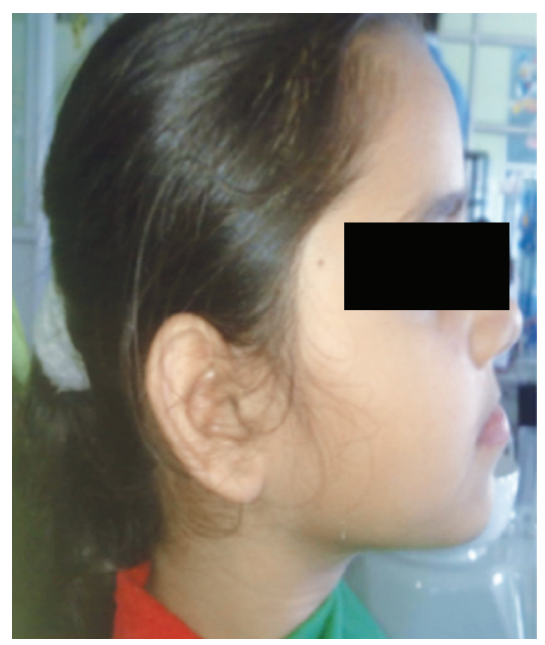

Fig. 1: Pretreatment profile of the patient

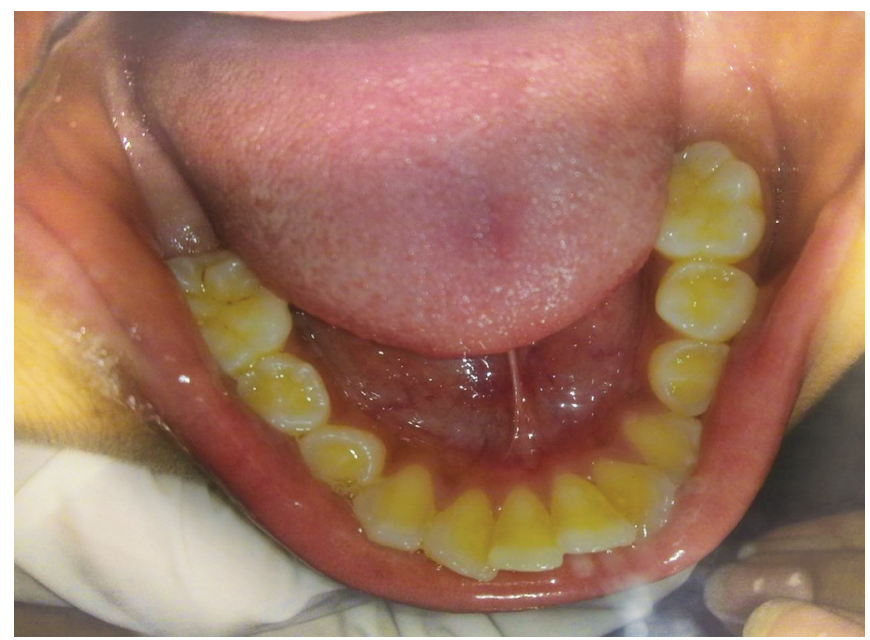

Fig. 3: Intraoral photograph of the mandibular arch with all complement of teeth present

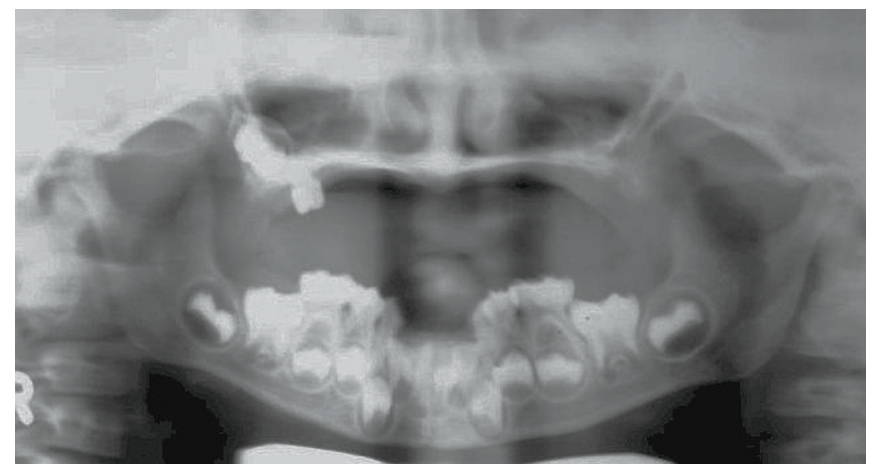

Fig. 5: Panoramic view at age 5 years

An analysis of the panoramic radiograph taken at the age of 5 years showed agenesis of all primary maxillary teeth except for an anomalous tooth 55 and developing tooth bud 16 (Fig. 5). Also, agenesis of primary mandibular incisors and presence of succeeding tooth buds were evident (Fig. 5). The present panoramic radiograph at age 12 years revealed complete agenesis of all maxillary teeth except for the presence of tooth 16,

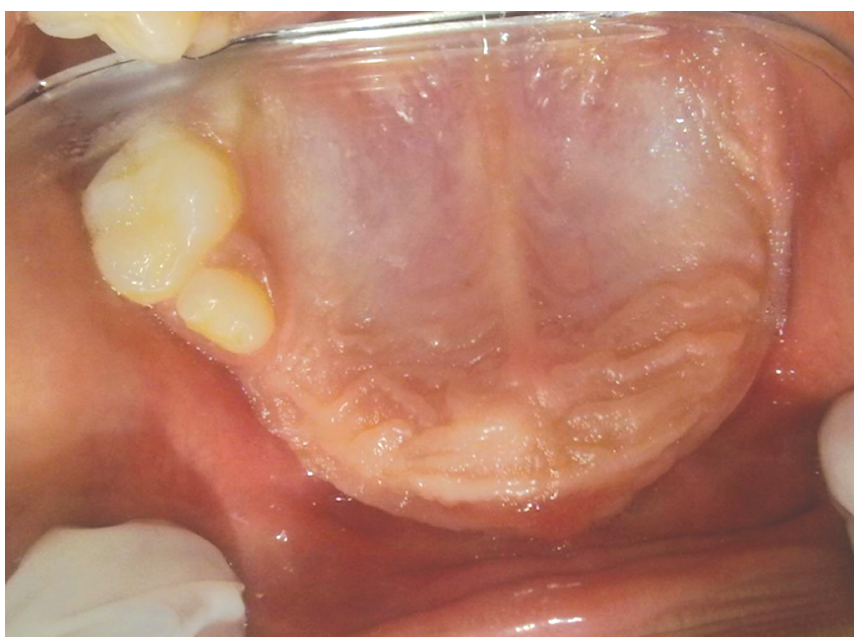

Fig. 2: Intraoral photograph of the maxillary arch showing missing permanent teeth except for 16 and an anomalous tooth 55

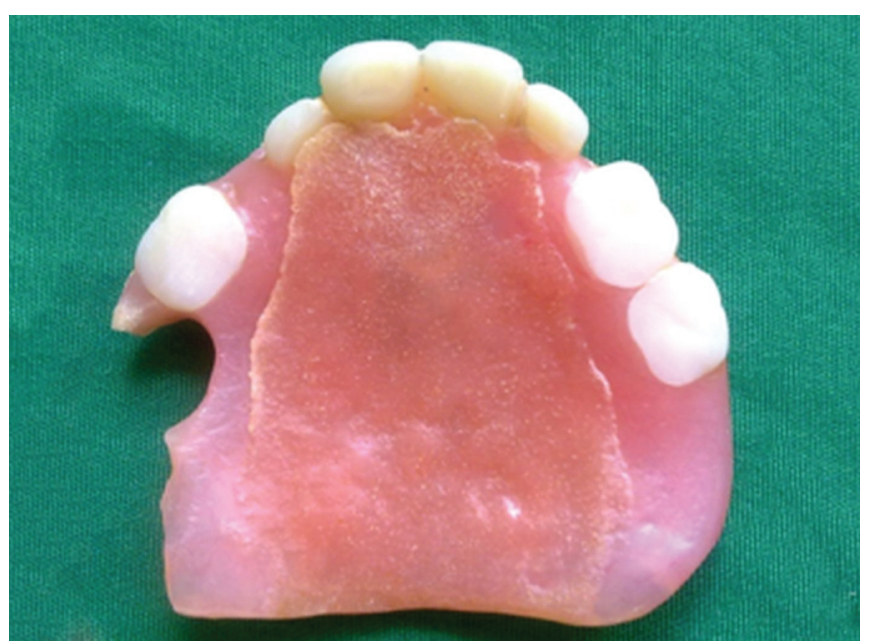

Fig. 4: Removable partial denture previously worn by the patient since the age of 5 years

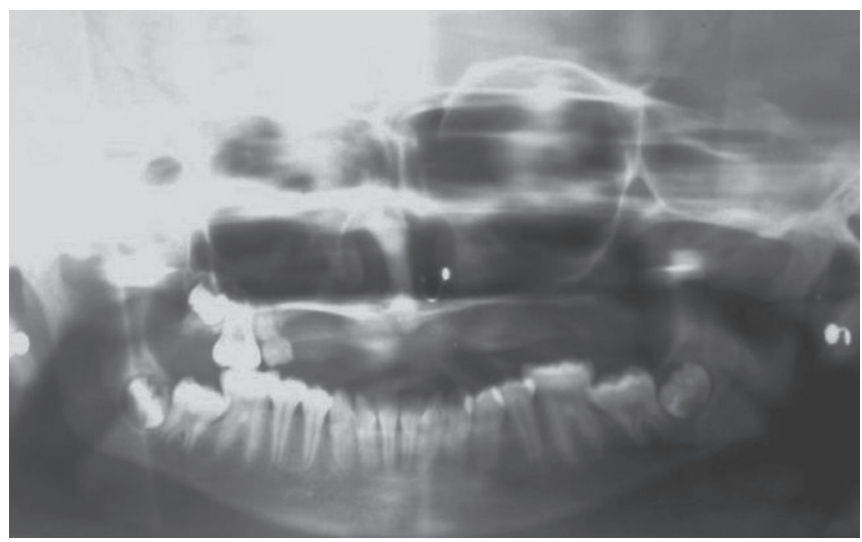

Fig. 6: Panoramic view at age 12 years

developing tooth 17 (permanent maxillary right second molar), and an anomalous tooth 55 (Fig. 6).

A referral to a pediatrician confirmed that there was no systemic or syndromic involvement. Blood investigation tests, thyroid function tests, and karyotyping performed were inconclusive. 


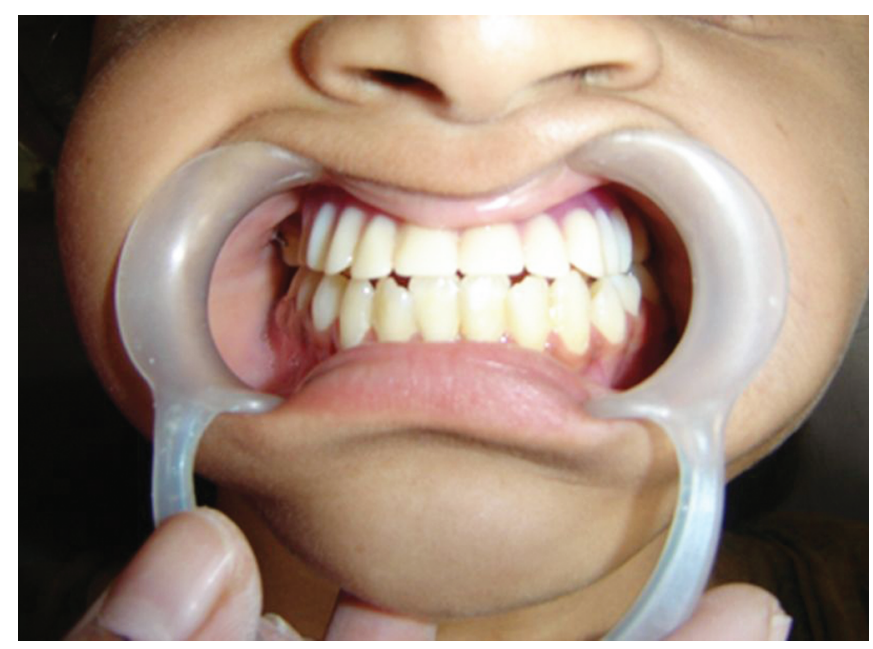

Fig. 7: Posttreatment view of the dentition

Absence of similar findings in maternal or paternal side of the family, parents, or siblings showed that a hereditary lineage was noticeably absent. A diagnosis of nonsyndromic oligodontia was made.

After careful evaluation and due consideration, a decision was taken to restore the functions and esthetics of the patient with a new removable maxillary denture. The previously worn denture was uncomfortable, impeding jaw growth and compromising mastication, speech, and esthetics. An alginate impression of the mandibular arch was taken. For the maxillary arch, an initial impression with impression compound was obtained. Maxillary custom tray was fabricated using autopolymerizing acrylic resin. Border molding was completed using green stick impression compound and secondary impression was made using zinc oxide eugenol impression paste. The maxillary-mandibular jaw relation was recorded. The occlusal rims established the vertical dimension, smile line, and midline of the face. These jaw relations were transferred to the articulator. A trial denture was fabricated and tested for esthetics, phonetics, and jaw relations. After satisfactory trial, processing of the denture was performed using heat cure acrylic resin through compression molding technique. The denture was inserted, and the patient guided regarding its usage, cleanliness, and maintenance with instructions to maintain oral hygiene. The child was thrilled with the new denture. Satisfactory occlusion, esthetics, and an improvement in facial profile was observed (Figs 7 and 8). The child was recalled the next day and subsequently put on regular follow-up visits.

The differential diagnosis includes ectodermal dysplasia, Rieger syndrome, and Van der Woude syndrome. The present condition differed from ectodermal dysplasia as the hair, nails, and sweating were normal. Absence of ocular features, such as glaucoma and craniofacial abnormalities, differentiated it from Rieger syndrome. Van der Woude syndrome was not considered because of an absence of cleft lip/palate, pits in the lower lip, and language or learning disabilities.

\section{Discussion}

Oligodontia is relatively a rare condition affecting $0.1-0.2 \%$ of the population. ${ }^{1}$ Oligodontia is classified as syndromic oligodontia or isolated oligodontia and may further, be familial or sporadic in nature. The inheritance pattern for nonsyndromic oligodontia can be autosomal (dominant or recessive) or X-linked with a

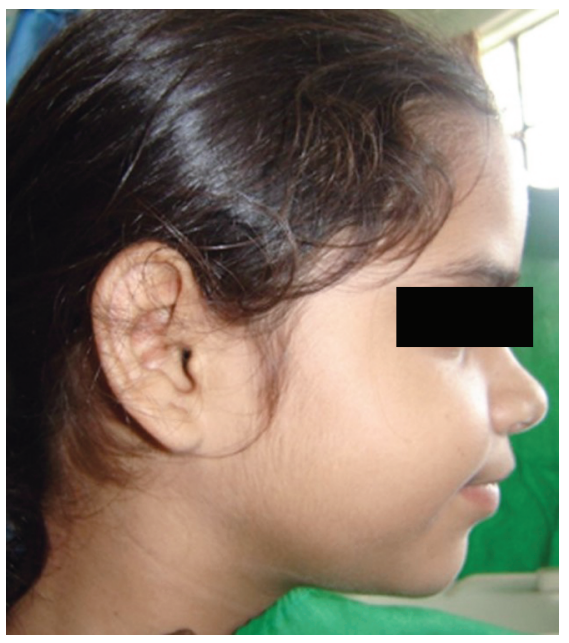

Fig. 8: Posttreatment profile view

considerable variation in penetrance and expressivity. ${ }^{7,8}$ Defects either in genes that encode transcription factors, such as MSX1 and PAX9, or those that encode for a protein involving Wnt signaling (AXIN2) and a transmembrane receptor of fibroblast growth factor, such as FGFR1, have been implicated in nonsyndromic tooth agenesis. ${ }^{5,6}$ The specific gene and the type of mutation in that gene determine the pattern of tooth agenesis. ${ }^{6}$ In the present case of nonsyndromic oligodontia involving a 12-year-old girl, there was a complete absence of maxillary teeth except for an erupting tooth 16 , a developing tooth 17 and an anomalous tooth 55 whereas the mandibular arch had a full complement of permanent teeth.

The differential diagnosis of syndromic and nonsyndromic form of oligodontia is made based on a thorough physical examination involving hair, nails, sweat glands, eyes, skeleton, and evaluation of any congenital disorder. ${ }^{7}$ For proper diagnosis and treatment planning, a clinical and radiographic examination inclusive of cephalometric analysis, orthopantomographic and intraoral radiographs along with study casts is required. ${ }^{2,9}$ In the present case, no associated syndrome/systemic involvement or a familial tendency was observed. The case was thus, that of non-syndromic isolated oligodontia.

The characteristic facial changes and growth disturbances observed in oligodontia are retrognathic and hypoplastic maxilla, retrognathic mandible with anterior rotation, reduced dimensions of alveolar process (both vertical and transverse), large inferior extension of maxillary sinus, receded midface, decreased anterior facial height, increased nasolabial angle, and a deep mentolabial sulcus. ${ }^{1}$ Delayed eruption of teeth with hampered growth of alveolar process, supraeruption of teeth opposing the missing teeth, impaction of primary molars, ectopic eruption, transposition, rotation, retention of primary teeth, pseudo diastema, or multiple diastema have also been reported. ${ }^{1,3}$ Occlusal disturbances include crossbite, deep overbite, and a steep inclination of maxillary incisors.'

Management of oligodontia in pediatric patients is challenging as it requires not only a knowledge of the restorative and prosthodontic techniques but also of growth and development and behavior management. ${ }^{10}$ The main objectives in dental rehabilitation are restoration of esthetics, prevention of psychosocial problems, restoration of masticatory function, prevention of space loss where there are erupted teeth, replacement of missing teeth 
without interfering with the growth of mandible and maxilla, and proper speech development. ${ }^{11}$

The critical factors in prosthetic rehabilitation are the number of missing teeth, their distribution in the dental arch, age of the patient along with expectation of the patients and parents. The treatment options in cases of fewer number of missing teeth are adhesive restorations, fixed prosthesis, and dental implants. ${ }^{3,12}$ In patients with many missing teeth, removable partial denture, overdentures, osseointegrated implants, and fixed prostheses can be considered. In children and adolescents due to anatomical changes related to facial growth, placement of implants is contraindicated and rather, should be deferred until after completion of the growth spurt or attainment of puberty. Growth of the jaws is impeded if implants are placed earlier which may act as "submerged" or ankylosed teeth with infraocclusion of restorations. Besides oligodontia patients, atrophy of the ridge is common, which contraindicates placement and compromises longevity of implants. Hence, implants should be avoided in growing maxilla until early adulthood. Conventional fixed prosthesis, when placed on young permanent teeth, entail considerable removal of tooth structure, risking pulp exposure because of thin enamel, dentin, a large coronal pulp, and high pulp horns. Additionally, an interference with jaw growth may result, which further contraindicates its use. Moreover, altered morphology of teeth is not favorable for retentive crown preparations.

Prosthetic rehabilitation in children should be carefully planned to accommodate changes in growth and development. Early prosthetic rehabilitation during primary dentition is temporary with a sole purpose of fulfilling esthetic, masticatory, and speech function. Subsequently, replacement of the prosthesis is required according to growth periods of the child.

The present case involved an oral rehabilitation of a 12-year-old child with nonsyndromic oligodontia. The treatment of choice was a removable partial denture to accommodate changes in growth, restore masticatory function and esthetics for the interim period.

\section{Conclusion}

The treatment protocol for oligodontia patients involves a fully integrated interdisciplinary team including a pediatrician, psychologist, geneticist, pediatric dentist, orthodontist, oral and maxillofacial surgeon, and a prosthodontist. However, role of a pediatric dentist is crucial when a young patient, during growth phase, needs an immediate but interim treatment, aiming at improvement of function, esthetics, and self-esteem.

\section{References}

1. Worsaae N, Jensen BN, Holm B, et al. Treatment of severe hypodontiaoligodontia-an interdisciplinary concept. Int J Oral Maxillofac Surg 2007;36(6):473-480. DOI: 10.1016/j.ijom.2007.01.021.

2. Ghazahfaruddin M, Mishra G, Haseebuddin S, et al. Oligodontia of permanent teeth: a rare case report. Indian J Stomatol 2011;2(4): 285-287.

3. Sevimay $M$, Akin C. Prosthetic treatment of nonsyndromic oligodontia. Eur J Prosthodont 2015;3(2):47-50. DOI: 10.4103/23474610.157842 .

4. Pemberton TJ, Das P, Patel PI. Hypodontia: genetics and future perspectives. Braz J Oral Sci 2005;4(13):695-706.

5. Hiremath MC. Nonsyndromic oligodontia: a rare case report. Arch Oral Sci Res 2012;2(2):103-107.

6. Chhabra N, Goswami M, Chhabra A. Genetic basis of dental agenesis-molecular genetics patterning clinical dentistry. Med Oral Patol Oral Cir Bucal 2014;19(2):e112-e119. DOI: 10.4317/medoral.19158.

7. Maganur PC, Satish V, Panda S, et al. Non-syndromic oligodontia: a rare case report. Austin J Dent 2017;4(6):1090.

8. Biradar VG, Hugar SI, Biradar SV. Oligodontia: a rare case report and literature review. Saudi J Health Sci 2013;2(2):127-129. DOI: 10.4103/2278-0521.117918.

9. Correia MF, Nogueira MN, Bedran TB, et al. Aesthetic rehabilitation of oligodontia in primary dentition with adhesive partial denture. Case Reports in Dentistry 2013;2013:872476. DOI: 10.1155/2013/872476.

10. Maroulakos G, Artopoulou II, Angelopoulou MV, et al. Removable partial dentures vs overdentures in children with ectodermal dysplasia: two case reports. Eur Arch Paediatr Dent 2016;17(3): 205-210. DOI: 10.1007/s40368-015-0212-x.

11. Gonçalves TM, Gonçalves LM, Sabino-Bezerra JR, et al.Multidisciplinary therapy of extensive oligodontia: a case report. Braz Dent J 2013;24(2):174-178. DOI: 10.1590/0103-6440201302158.

12. Muretic Z, Magdalenic-Mestrovic M, Zarkovic D. An interdisciplinary approach to the treatment of oligodontia. Acta Stomat Croat 2001. 117-120. 